\title{
GMR
}

\section{Y-STR INRA189 polymorphisms in Chinese yak breeds}

\author{
Z.J. Ma',2, S.M. Chen², Y.G. Sun ${ }^{2}$, Y.L. Xi' ${ }^{1}$, R.Z. Li' ${ }^{2}$, J.T. $\mathrm{Xu}^{2}$ and C.Z. Lei ${ }^{1}$ \\ ${ }^{1}$ College of Animal Science and Technology, Northwest A\&F University, \\ Yangling, China \\ ${ }^{2}$ Qinghai Academy of Animal Science and Veterinary Medicine, Qinghai University, \\ Xining, China \\ Corresponding author: C.Z. Lei \\ E-mail: leichuzhao1118@126.com / zhijiema@126.com
}

Genet. Mol. Res. 14 (4): 18859-18862 (2015)

Received September 10, 2015

Accepted November 16, 2015

Published December 28, 2015

DOI http://dx.doi.org/10.4238/2015.December.28.35

ABSTRACT. To further explore Y-STR INRA189 polymorphisms in the yak, and to determine the genetic differences among yak breeds, genotyping analysis of INRA189 in 102 male yak individuals from three yak breeds in Qinghai Province of China was performed. Genotyping revealed the presence of four alleles, with sizes of 149,155, 157, and $159 \mathrm{bp}$, respectively. Of these, the 157-bp allele, which was found with the highest frequency in the three yak breeds, was the dominant allele. Interestingly, the 149-bp allele was only detected in the Gaoyuan breed, and the 159-bp allele was only found in the Huanhu and Datong breeds. Only the 157- and 155-bp alleles were found in all three yak breeds. Taking the three yak breeds as a single population, the frequency of these four alleles was $0.0294,0.0686$, 0.8628 , and 0.0392 , respectively. The average polymorphism information content in the three yak breeds was 0.2379 , indicating that the INRA189 was a low polymorphic Y-STR marker in yak.

Key words: Yak; Polymorphism; Y-STR; INRA189 


\section{INTRODUCTION}

Yak (Bos grunniens) is a bovine species that lives on the Qinghai-Tibetan Plateau and its adjacent territories, which are at high altitudes $(2000-5000 \mathrm{~m})$ and are characterized by low atmospheric oxygen and low temperatures. This species can provide pastoralists and agropastoralists living in these areas, mainly the Tibetans, with products (milk, meat, hair, and hides, and manure for fuel) and services (draught, packing, and riding) (Wiener et al., 2003). In China, there are 12 officially recognized yak breeds and around 14 million individuals (Wiener et al., 2003). In Qinghai province, there are three yak breeds, including two indigenous breeds (Gaoyuan and Huanhu) and one improved breed (Datong). Currently, there have been few studies on Y-chromosome markers in the yak ( $\mathrm{Li}$ et al., 2014). Previous genetic analyses indicated that INRA189 is a yak Y-chromosome-specific microsatellite (Y-STR), which has 2-3 alleles in partial Chinese yak populations and in the Swiss yak population (Han et al., 2000; Nguyen et al., 2005). However, until now, no information on the INRA189 marker is available that can be used to characterize genetic differences between the three yak breeds in Qinghai. The principal aims of this study were to characterize Y-STR INRA189 marker polymorphisms in three yak breeds from Qinghai province and to determine paternal genetic differences.

\section{MATERIAL AND METHODS}

\section{Primer sequence}

A pair of primers was employed as previously described (Kappes et al., 1997). The forward primer was labeled at the $5^{\prime}$ end with HEX fluorescent dye (Sangon, Shanghai, China). The sequences were as follows: Forward primer: 5'-TTT TGT TTC CCG TGC TGA G-3'; Reverse primer: 5'-GAA CCT CGT CTC CTT GTA GCC-3'.

\section{Sampling, DNA extraction, and polymerase chain reaction (PCR) conditions}

A total of 102 male individuals, comprising 34 Gaoyuan (GY) yaks collected in Qumalai county, 29 Huanhu $(\mathrm{HH})$ yaks collected in Gonghe county, and 39 Datong (DT) yaks collected at the Datong yak breeding farm of Qinghai province, China, were used for INRA189 genotype analysis. In addition, 10 female Gaoyuan (GY) yaks obtained from the same sampling sites were used as negative controls. Genomic DNA was extracted from blood using a phenol-chloroform protocol followed by an ethanol precipitation step. The PCR reaction mixture contained 50-100 ng yak genomic DNA, $10 \mu \mathrm{M}$ each primer, 2X Multiplex PCR Master Mix (Qiagen, Shanghai, China) and $\mathrm{ddH}_{2} \mathrm{O}$ to a final volume of $10 \mu \mathrm{L}$. The amplification conditions were as follows: $95^{\circ} \mathrm{C} / 5 \mathrm{~min}$, followed by 35 cycles at $94^{\circ} \mathrm{C} / 30 \mathrm{~s}, 60^{\circ} \mathrm{C} / 1 \mathrm{~min}, 72^{\circ} \mathrm{C} / 30 \mathrm{~s}$, and final synthesis at $68^{\circ} \mathrm{C} / 10 \mathrm{~min}$.

\section{Genotyping and statistical analysis}

Amplified products were scanned together with the GS-500 Liz size standard (Sangon, Shanghai, China) in an ABI 3730xI DNA Analyzer (Applied Biosystems) and fragment lengths were scored using GeneMarker v1.97 (http://www.softgenetics.com/GeneMarker.html). Genotypes were assigned for each individual based on allele size data. Allele frequencies were estimated using the GENETIX 4.05 software package (Belkhir et al., 2004). The polymorphic information 
content (PIC) was calculated using the PIC_CALC 0.1 software (Nagy et al., 2012) to estimate the informativeness of the microsatellite marker.

\section{RESULTS AND DISCUSSION}

In our study, the INRA189 marker was only identified in male yaks, which is consistent with the results of previous studies (Han et al., 2000; Nguyen et al., 2005). These data indicate that INRA189 is a yak Y-STR marker, which can be used in future studies to characterize malemediated genetic events, paternal genetic diversity, and the origin and evolution of yak breeds.

Previously, Han et al. (2000) and Nguyen et al. (2005) indicated that there are 2-3 INRA189 alleles in partial yak populations. However, in the present study, the results obtained by genotyping INRA189 in 102 male yak individuals revealed the presence of four alleles, with sizes of $149,155,157$, and $159 \mathrm{bp}$, respectively (Figure 1). Of these, the allele containing $157 \mathrm{bp}$ was found at the highest frequency in the three yak breeds studied, and is therefore the dominant allele. Interestingly, the allele containing 149 bp was only detected in the Gaoyuan breed, and the 159-bp allele was only found in the Huanhu and Datong breeds. Only the 157- and 155-bp alleles were identified in all three yak breeds. Taking the three yak breeds as a single population, the frequency of these four alleles was $0.0294,0.0686,0.8628$, and 0.0392 (Table 1), respectively. The PIC in the three yak breeds was 0.2379 , which indicates that the INRA189 locus has low PIC (PIC <0.25) and is therefore a lower polymorphic Y-STR marker in the yak.
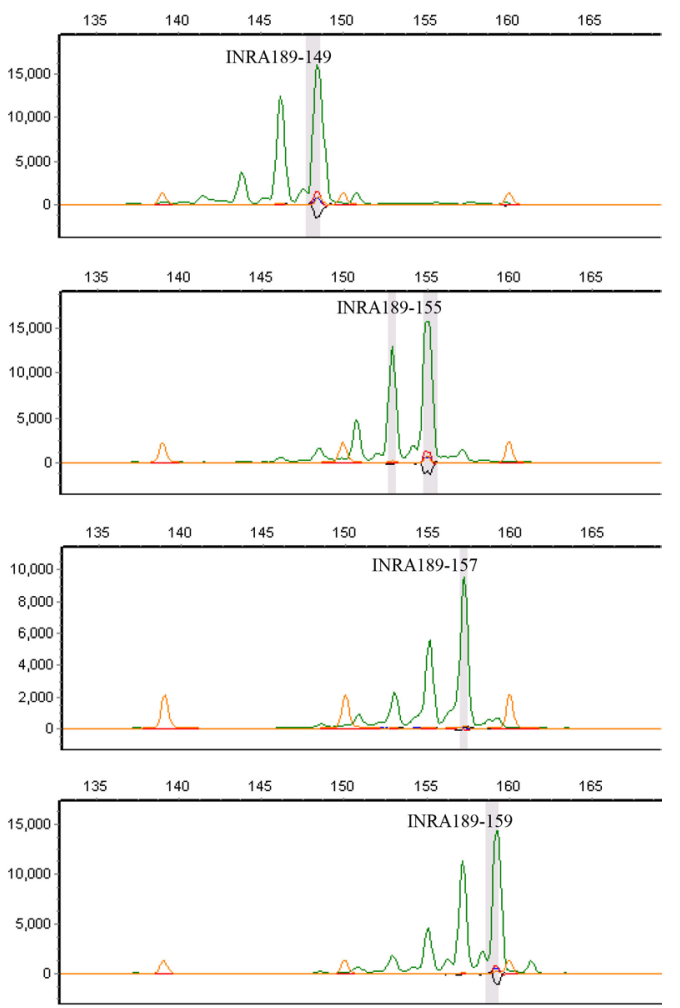

Figure 1. Different alleles of INRA189 in three yak breeds. 
Table 1. Allele frequencies of the polymorphic INRA189 marker in three yak breeds.

\begin{tabular}{lccccc}
\hline Breed & Sample No. & \multicolumn{3}{c}{ INRA189 } \\
\cline { 2 - 6 } & & 149 & 155 & 157 & $0.8824(30)$ \\
\hline Gaoyuan & 34 & $0.0882(3)$ & $0.0294(1)$ & $0.8974(35)$ & $0(0)$ \\
Datong & 39 & $0(0)$ & $0.0513(2)$ & $0.7931(23)$ & $0.0513(2)$ \\
Huanhu & 29 & $0(0)$ & $0.1379(4)$ & $0.8628(88)$ & $0.0690(2)$ \\
Total & 102 & $0.0294(3)$ & $0.0686(7)$ & $0392(4)$ \\
\hline
\end{tabular}

Numbers in brackets represent the numbers of individuals with each allele.

\section{Conflicts of interest}

The authors declare no conflict of interest.

\section{ACKNOWLEDGMENTS}

Research supported by the National Natural Science Foundation of China (\#31360267), the "123" high-level personnel training project of Qinghai University, the Program of National Beef Cattle Industrial Technology System (\#CARS-38), the Science \& Technology Department project of Qinghai Province (\#2015-ZJ-712), the International Scientific and Technological Cooperation project of the National Science and Technology Ministry (\#2013DFA31420), and the scientific and technological innovation platform of the bovine (milk, meat, wool) industry of Qinghai Province.

\section{REFERENCES}

Belkhir K, Borsa P, Chikhi L, Raufaste N, et al. (2004). GENETIX 4.05, logiciel sous Windows TM pour la génétique des populations. Laboratoire Génome, Populations, Interactions, CNRS UMR 5000, Université de Montpellier II, Montpellier.

Han JL, Ochieng JW, Rege JEO and Hanotte O (2000). Low level of cattle introgression in yak populations from Bhutan and China: Evidences from Y-specific microsatellites and mitochondrial DNA markers. Proceedings of the 3rd International Congress on Yak, Lhasa, 190-196.

Kappes SM, Keele JW, Stone RT, McGraw RA, et al. (1997). A second generation linkage map of the bovine genome. Genome Res. 7: 235-249.

Li R, Wang SQ, Xu SY, Huang JP, et al. (2014). Novel Y-chromosome polymorphisms in Chinese domestic yak. Anim. Genet. 45: 449-452.

Nagy S, Poczai P, Cernák I, Gorji AM, et al. (2012). PICcalc: an online program to calculate polymorphic information content for molecular genetic studies. Biochem. Genet. 50: 670-672.

Nguyen TT, Genini S, Ménétrey F, Malek M, et al. (2005). Application of bovine microsatellite markers for genetic diversity analysis of Swiss yak (Poephagus grunniens). Anim. Genet. 36: 484-489.

Wiener G, Han JL and Long RJ (2003). The Yak. 2nd edn. Regional Office for Asia and the Pacific of the Food and Agriculture Organization of the United Nations, Bangkok. 